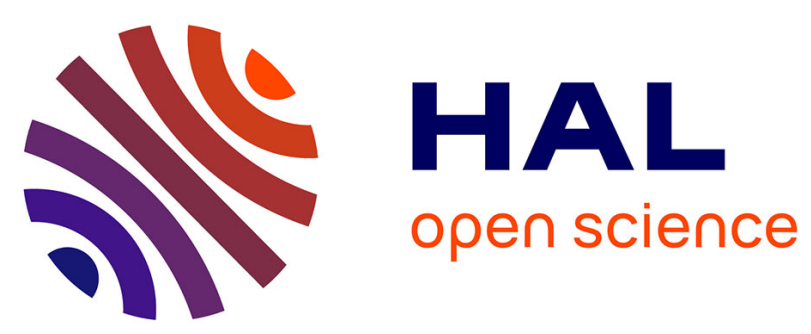

\title{
Phase-field investigation of the coarsening of porous structures by surface diffusion
}

Pierre-Antoine Geslin, Mickael Buchet, Takeshi Wada, Hidemi Kato

\section{To cite this version:}

Pierre-Antoine Geslin, Mickael Buchet, Takeshi Wada, Hidemi Kato. Phase-field investigation of the coarsening of porous structures by surface diffusion. Physical Review Materials, 2019, 3 (8), 10.1103/PhysRevMaterials.3.083401 . hal-02268976

\section{HAL Id: hal-02268976 https://hal.science/hal-02268976}

Submitted on 22 Aug 2019

HAL is a multi-disciplinary open access archive for the deposit and dissemination of scientific research documents, whether they are published or not. The documents may come from teaching and research institutions in France or abroad, or from public or private research centers.
L'archive ouverte pluridisciplinaire HAL, est destinée au dépôt et à la diffusion de documents scientifiques de niveau recherche, publiés ou non, émanant des établissements d'enseignement et de recherche français ou étrangers, des laboratoires publics ou privés. 


\title{
Phase-field investigation of the coarsening of porous structures by surface diffusion
}

\author{
Pierre-Antoine Geslin $\mathbb{1}^{*}$ \\ Univ. Lyon, INSA-Lyon, UCBL, UMR CNRS 5510, MATEIS, F69621 Villeurbanne Cedex, France \\ and ELyTMAX UMI 3757, CNRS - Université de Lyon - Tohoku University, International Joint Unit, Tohoku University, Sendai, Japan \\ Mickaël Buchet \\ WPI-AIMR, Tohoku University, 2-1-1 Katahira, Aobaku, Sendai 980-0812, Japan \\ and Institue of Geometry, Graz University of Technology, Kopernikusgasse 24, 8010 Graz, Austria \\ Takeshi Wada and Hidemi Kato \\ Institute for Materials Research, Tohoku University, 2-1-1 Katahira, Aobaku, Sendai 980-8577, Japan
}

(Received 24 May 2019; published 8 August 2019)

\begin{abstract}
Nano and microporous connected structures have attracted increasing attention in the past decades due to their high surface area, presenting interesting properties for a number of applications. These structures generally coarsen by surface diffusion, leading to an enlargement of the structure characteristic length scale. We propose to study this coarsening behavior using a phase-field model for surface diffusion. In addition to reproducing the expected scaling law, our simulations enable to investigate precisely the evolution of the topological and morphological characteristics along the coarsening process. In particular, we show that after a transient regime, the coarsening is self-similar as exhibited by the evolution of both morphological and topological features. In addition, the influence of surface anisotropy is discussed and comparisons with experimental tomographic observations are presented.
\end{abstract}

DOI: 10.1103/PhysRevMaterials.3.083401

\section{INTRODUCTION}

Finely porous materials are characterized by a complex structure of ligaments. Because the size of these ligaments can reach very small scales (down to a few nanometers for nanoporous gold [1]), they display a very large surface area that is attractive for a number of applications, ranging from actuators [2,3] to catalyst materials [4,5]. It was also suggested that the nanoscale ligaments could lead to interesting mechanical properties due to the confinement of dislocation activity [6,7], or be used as radiation resistant materials [8], because of the fast annihilation of point defects at the interface.

These porous structures can be obtained from various methods. Eletrochemical dealloying enables to produce very fine nanoporous structures but is limited to noble metals such as gold, palladium or copper [1,9-11]. Recently, this limitation has been overcome with the development of liquid metal dealloying, that consists in using a molten metal to selectively dissolve one component out of a binary alloy [12]. This processing technique presents the advantage of being applicable to a wide range of metals including titanium [13], iron [14], niobium [15], and tantalum [16,17], or semiconductors such as silicon [18]. Also, comparable porous structures have been obtained from vapor phase dealloying that relies on the low partial pressure of one of the component of the alloy to trigger selective dissolution [19,20].

\footnotetext{
*pierre-antoine.geslin@insa-lyon.fr
}

Despite the differences between these processing routes, the resulting materials present very similar microstructures that evolve at least partly through surface diffusion as demonstrated by the activation energy of the coarsening process and by the expected power-law evolution of the ligament size [13,20-22]. This coarsening process is important to control and limit as much as possible because it leads to a severe decrease of the specific surface area and therefore to a drop of materials properties. Common strategies consist of decreasing the surface diffusion by reducing the dealloying temperature $[15,17,23]$ or adding impurities that reduce surface diffusivity [24,25].

The coarsening behavior of these microstructures has been investigated previously with both experimental and numerical methods. Especially, nanotomography experiments demonstrated that nanoporous gold coarsens by surface diffusion and that its morphology is significantly affected by surface energy anisotropy [26,27] but raised the question of the selfsimilar character of this coarsening. Using atomistic Monte Carlo simulations [28], Erlebacher showed that the coarsening mechanism of finite-size dealloyed particles does not follow a self-similar behavior and attributes this to topological changes during coarsening. Another atomistic study [29] based on molecular dynamics reports that plasticity can play an important role in the network reconstruction during the coarsening process of ligaments of a few nanometers.

On the other hand, phase-field modeling is an attractive tool to investigate this type of complex evolution. As a continuous approach, it can be used to model arbitrary length and time scales in contrast with atomistic methods. Furthermore, 
the phase-field approach deals naturally with complex topological evolution as encountered during these microstructural evolutions. Finally, it allows a better control of the surface energy anisotropy than atomistic models where the anisotropy is function of the choice of the interatomic potential. The phasefield approach has been successfully applied to investigate the evolution of structures obtained after spinodal decomposition and their coarsening by volume diffusion [30-32]. These studies shed light on this coarsening behavior, showing that these structures follow the appropriate power law expected for bulkdiffusion surface relaxation [33]. In addition, it was shown that the morphological and topological characteristics of these structures evolve in a self-similar way. We note also that several previous phase-field studies discussed microstructural evolution by surface diffusion $[34,35]$, but these studies being limited to 2D geometry, it is hazardous to generalize their findings to 3D: in particular, the influence of the topological changes can only be investigated in $3 \mathrm{D}$.

Therefore it is highly desirable to use a similar continuous phase-field approach to investigate microstructual evolution by surface diffusion in 3D. The topological and morphological evolution obtained by surface diffusion could be significantly different than the one obtained by volume diffusion [30-32]. Indeed, as pointed out by Erlebacher [28], in the surface diffusion case, the support on which the diffusion takes place is affected by topological changes such as ligament pinch-off events that break diffusion paths between regions. In this paper, we propose to use a phase-field model for surface diffusion to investigate in details this coarsening process. The paper is structured as follows: first, the model and its numerical implementation are described in Sec. II. Then, the coarsening dynamics and the self-similar character of the surface evolution are discussed in Sec. III and the influence of surface anisotropy is discussed in Sec. IV. Finally, a valuable comparison with experimental results is presented in Sec. V.

\section{PHASE-FIELD MODEL FOR SURFACE DIFFUSION}

In the past decades, phase-field modeling has emerged as a tool of choice to investigate free boundary problems involving complex morphological and topological changes, as the one presently at hand. It relies on the introduction of a phase-field denoted $\phi$ which takes different values in different phases and adopts a smooth variation at the interface location. The first models proposed to investigate surface diffusion [35,36] simply by considering a CahnHilliard dynamics with a phase-dependent mobility that vanishes in both phases and is nonzero at the interface. Such simple formulation however converges poorly towards the expected sharp-interface behavior as shown in Refs. [37,38], where the inaccuracies of this approach are pointed out. Lee et al. [38] showed that employing a double-obstacle potential instead of the classical double-well can however improve the situation.

The model developed by Rätz, Ribalta, and Voigt [39]— hereafter referred as the RRV model-has been shown to converge faster towards the appropriate sharp interface behavior in the limit of vanishing interface thickness [37,39]. For an isotropic surface energy and mobility, the RRV model relies on the following time-dependent equations for the phase-field $\phi$ :

$$
\begin{aligned}
\frac{\partial \phi}{\partial t} & =-\nabla \cdot \boldsymbol{J}, \\
\boldsymbol{J} & =-\frac{12 M f(\phi)}{W^{2}} \nabla \mu, \\
\mu & =\frac{-W^{2} \nabla^{2} \phi+2 f^{\prime}(\phi)}{10 f(\phi)},
\end{aligned}
$$

where $M$ is an effective kinetic coefficient, $W$ denotes the interface width, $\boldsymbol{J}$ is the flux, $\mu$ is the chemical potential and $f(\phi)=\phi^{2}(1-\phi)^{2}$ is the double-well function commonly used in phase-field approaches. It was noted in Ref. [37] that the term $f(\phi)$ in Eq. (3) combines adequately with the decay of the mobility function to improve the convergence towards the appropriate sharp interface limit.

We note that the kinetic coefficient $M$ can be expressed as a function of physical parameters $[40,41]$ and scales like the product of the surface energy $\gamma$ by the surface diffusion coefficient $D_{s}$ :

$$
M=\frac{D_{s} \gamma N_{s} \Omega^{2}}{k T},
$$

where $N_{s}$ is the number of diffusing atoms per surface area, $\Omega$ is the atomic volume, and $k T$ has its usual meaning. The coefficient $M$ can in principle be estimated from molecular dynamics simulations for a specific interface. However, in this study, the specific choice of $M$ remains unimportant because it is used to rescale the unique timescale of the problem at hand.

The time integration of Eqs. (1)-(3) is performed after discretization of $\phi$ on a finite difference grid of dimensions $\left(N_{x}, N_{y}, N_{z}\right)$. To stabilize the numerical scheme and increase the computational efficiency, we use a semi-implicit scheme inspired from Ref. [35], that relies on the evaluation of $\phi$ and the fluxes $\boldsymbol{J}$ in Fourier space. After the Fourier transform of the discretized phase-field $\phi$ (denoted $\tilde{\phi}=\mathcal{F}(\phi))$, its second derivative $\nabla^{2} \phi$ appearing in Eq. (3) can easily be estimated in Fourier space with a multiplication by a linear operator $\tilde{L}$ that performs second-order derivatives finite differences:

$$
\begin{aligned}
& \left\{\mathcal{F}\left(\nabla^{2} \phi\right)\right\}_{\tilde{n}_{x}, \tilde{n}_{y}, \tilde{n}_{z}}=\tilde{L}_{\tilde{n}_{x}, \tilde{n}_{y}, \tilde{n}_{z}} \tilde{\phi}_{\tilde{n}_{x}, \tilde{n}_{y}, \tilde{n}_{z},} \\
& \tilde{L}_{\tilde{n}_{x}, \tilde{n}_{y}, \tilde{n}_{z}}=\left[\sum_{v=x, y, z} \frac{2}{\Delta v^{2}} \cos \left(\frac{2 \pi \tilde{n}_{v}}{N_{v}}\right)\right],
\end{aligned}
$$

where $\left(\tilde{n}_{x}, \tilde{n}_{y}, \tilde{n}_{z}\right)$ are the indices on the Fourier space grid and $\Delta x, \Delta y, \Delta z$ denote the grid spacing in the different spacial directions.

The next step consists in going back to real space and evaluating the chemical potentials $\mu$ and the fluxes $\boldsymbol{J}$ from finite differences before performing a Fourier transform to obtain $\tilde{\boldsymbol{J}}$ in Fourier space.

Finally, the Fick equation (1) is integrated in Fourier space:

$$
\frac{\tilde{\phi}^{t+\Delta t}-\tilde{\phi}^{t}}{\Delta t}=\tilde{\boldsymbol{D}} \cdot \tilde{\boldsymbol{J}}
$$

where $\Delta t$ denotes the time-step and $\tilde{D}_{\tilde{n}_{x}, \tilde{n}_{y}, \tilde{n}_{z}}^{v}=\frac{i}{\Delta \nu} \sin \left(\frac{2 \pi v}{N_{v}}\right)$ is a linear operator that performs centered finite differences. As noted in Ref. [35], integrating this equation with a forward Euler scheme leads to a very severe time-step constraint to assure numerical stability due to the fourth order 
derivative of $\phi$. To overcome this limitation and stabilize the numerical scheme, Zhu et al. [35] propose to add the term $A\left(\tilde{L}^{2} \tilde{\phi}^{t+\Delta t}-\tilde{L}^{2} \tilde{\phi}^{t}\right)$ to Eq. (7). For $\Delta t$ small enough, this additional term has little influence on the dynamics but leads to the following semi-implicit scheme:

$$
\tilde{\phi}^{t+\Delta t}=\tilde{\phi}^{t}+\frac{1}{1+A \tilde{L}^{2}} \tilde{\boldsymbol{D}} \cdot \tilde{\boldsymbol{J}} .
$$

Following Ref. [35], we choose a coefficient $A=1 / 2$, allowing an excellent stabilization of the numerical scheme while limiting integration errors.

In the following, we will consider an interface width $W=2 \Delta x, \Delta x$ being the grid spacing considered equal in all three directions. Before numerical integration, all the lengths and times are rescaled by $W$ and $W^{4} / M$, respectively. Also, a time step $d t=0.02 \mathrm{~W}^{4} / M$ is considered in the following.

The numerical implementation of the model is checked by comparing the relaxation dynamics of a slightly perturbed interface to the sharp interface analytical solution; the results are summarized in Appendix A.

\section{COARSENING KINETICS AND SELF-SIMILARITY}

\section{A. Choice of the initial microstructure}

To generate numerical microstructures similar to the bicontinuous structures obtained from dealloying experiments, we use a phase-field model for spinodal decomposition, as the one described in Ref. [31]. From a random noise distribution, a bicontinuous microstructure naturally emerges for volume fractions in the range $37.5 \%-62.5 \%$. For volume fractions lower than $35 \%$ and higher than $65 \%$, one of the phase is not continuous, as noted in Ref. [32]. In the case of surface diffusion, the coarsening behavior is greatly affected by the connectivity of the phase since disconnected elements do not interact with the rest of the microstructure. Therefore we discard these configurations and restrict ourselves to the connected microstructures. We note also that, for obvious reasons, the microstructure evolution is symmetric with respect to the $50 \%$ composition; therefore, we can restrict ourselves to the composition range $37.5 \%-50 \%$. The size of the microstructure features can be controlled by interrupting the spinodal decomposition at various times. In our case, the initial characteristic length scale is chosen small compared to the system size in order to have a statistically representative structure and large compared to the interface width to avoid any spurious interface effect. In practice, we have $12 W \lesssim \lambda \lesssim L / 8$. A typical initial microstructure is depicted in Fig. 1(a).

The structures obtained from spinodal decompositions are then used as input files to the surface-diffusion model presented above. In the following, we will consider results for four different volume fractions $37.5 \%, 40 \%, 45 \%$, and $50 \%$.

\section{B. Morphological evolution}

The first morphological quantity of interest is the characteristic length scale of the microstructure that will be denoted $\lambda$. Various definitions of such a characteristic length scale can be considered and are in principle equivalent: most authors [26,27,30-32] use the inverse of the specific surface area $\lambda_{S}=V / S$ as a measure of the characteristic length scale.
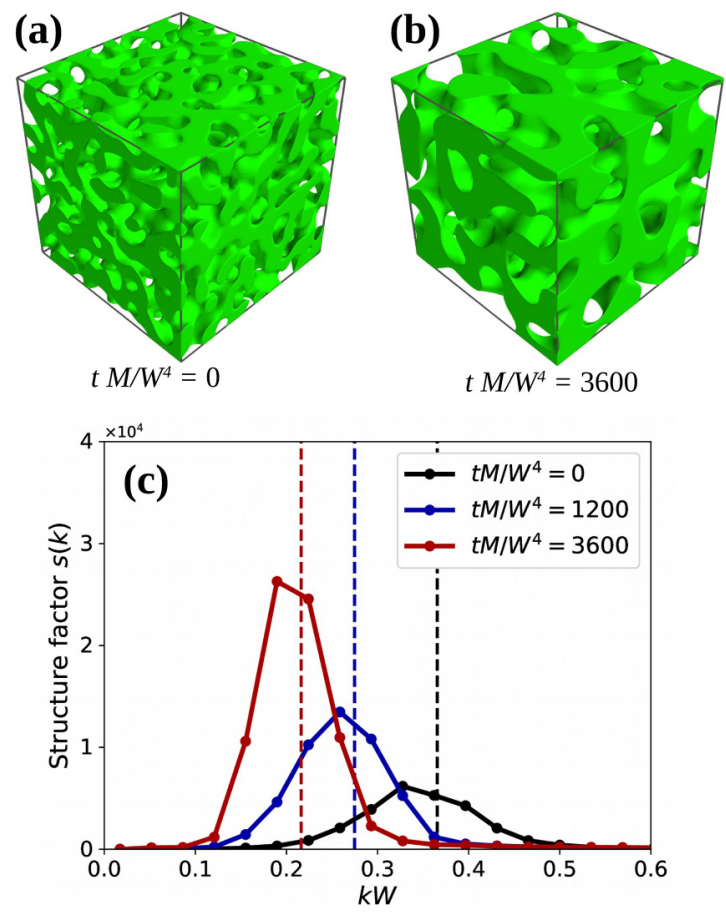

FIG. 1. (a) Example of initial microstructure considered for volume fraction $\rho=50 \%$. (b) Coarsened structure obtained at $t M / W^{4}=3600$. (c) Spherically averaged structure factors obtained from three different configurations along a simulation and the corresponding values of $k_{0}$ shown with dash lines (see text for explanations).

In this work, we prefer to use a definition of the length scale that is related to the size of the elementary cell of the microstructure. We rely on previous work $[35,42]$ to estimate this characteristic length scale from the structure factor of the microstructure defined as

$$
s(\boldsymbol{k})=\frac{\sum_{\boldsymbol{r}} \sum_{\boldsymbol{r}^{\prime}} e^{-i \boldsymbol{k} \cdot \boldsymbol{r}}\left[\phi\left(\boldsymbol{r}+\boldsymbol{r}^{\prime}\right) \phi(\boldsymbol{r})-\langle\phi\rangle^{2}\right]}{N^{2}\left(\left\langle\phi^{2}\right\rangle-\langle\phi\rangle^{2}\right)},
$$

where $N=N_{x} N_{y} N_{z}$ is the number of grid points of the domain and the sums over $r$ and $r^{\prime}$ run on the 3D real space grid. Considering that the structure does not have any privileged direction and is isotropic, we then perform a spherical average in $\boldsymbol{k}$ space to obtain the data shown in Fig. 1(c). We clearly see that the averaged structure factor presents a peak-value corresponding to a characteristic wave vector $k_{0}$. Because the maximum of this distribution may be difficult to determine precisely due to the effect of the underlying discrete grid, we compute the first moment of the structure factor and obtain an estimate of $k_{0}$ represented with dash lines in Fig. 1(c). The characteristic length scale is then defined as $\lambda=2 \pi / k_{0}$ and represents the size of the elementary cell of the structure, or in other words, the average distance between the center of two neighboring ligaments.

Let us note that this definition of the cell size is rather robust with respect to changes of the volume fraction. Indeed, if we consider a given structure and erode the ligaments to reduce the volume fraction, the surface area is reduced as well, and the characteristic length scale $\lambda_{S}$ increases, while our definition of $\lambda$ remains unchanged, making possible to easily compare structures of different volume fractions. In 

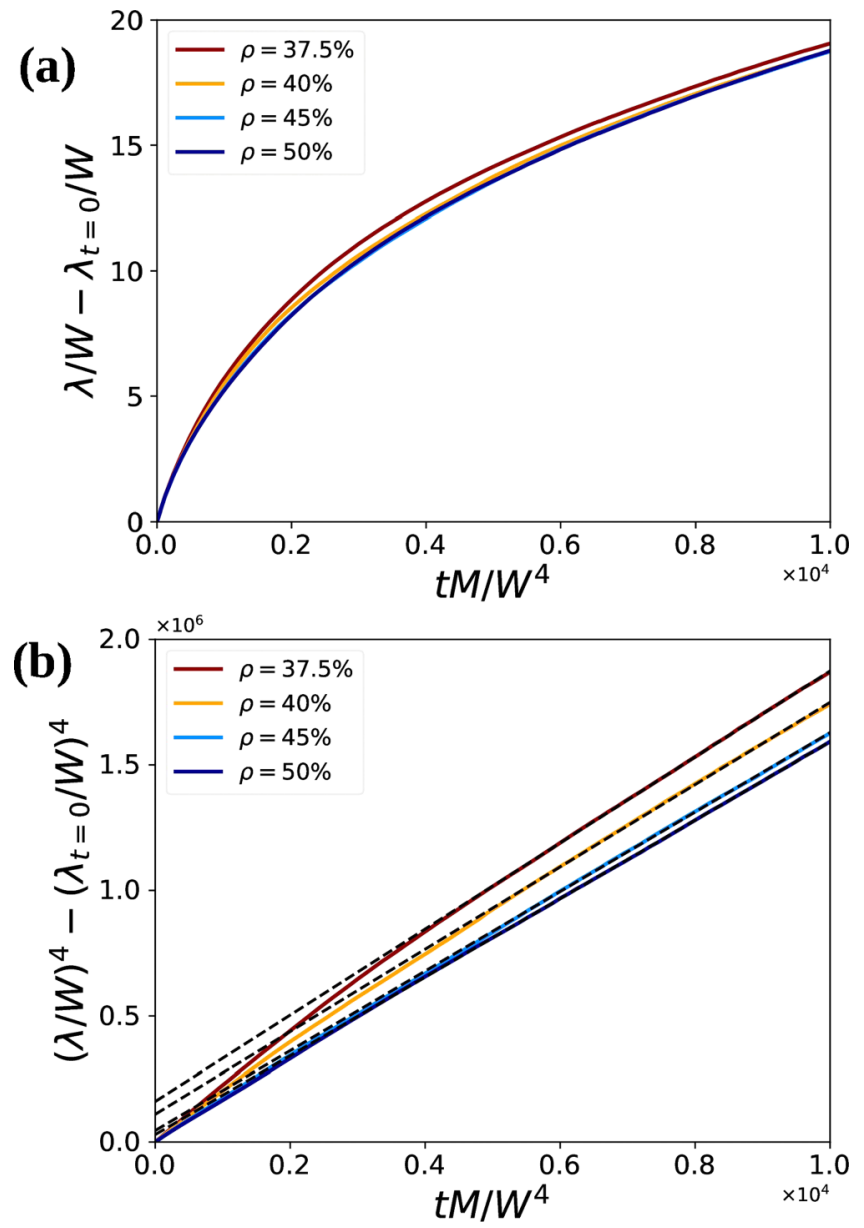

FIG. 2. (a) Evolution of the characteristic length scale as function of simulation time obtained for various volume fractions. (b) Same graph with the $y$ axis elevated to the fourth power.

addition, our definition of $\lambda$ is not sensitive to the details of the surface: surfaces obtained from experimental pictures or atomistic calculations may include a roughness coming from experimental noise or thermal fluctuations that may increase spuriously the value of $S$ (and therefore decrease the estimate of $\lambda_{S}$ ). These effects are also avoided when using our definition of $\lambda$ as the cell size because it does not depends on the details of the interface.

With this definition of the characteristic length scale, the time evolution $\lambda(t)$ can be compared between different volume fractions as shown on Fig. 2(a). These curves are obtained from averaging ten different simulations to ensure statistical significance of our results. Figure 2(a) shows that the coarsening rate depends slightly on the volume fraction. To better display the discrepancy between the different volume fractions, we show in Fig. 2(b) the time evolution of the length scale elevated to the fourth power. The curves obtained for $\rho=50 \%$ and $45 \%$ follow a perfect line, showing that they coarsen with a power law $\lambda^{4} \sim t$, expected for surface diffusion evolution [33]. However, for lower volume fractions ( $\rho=40 \%$ and $37.5 \%$ ), we clearly see a slight deviation from this power-law behavior, the curves being slightly concave. This is attributed to the development of a transient regime in the first part of the dynamics. After this transient regime, the
TABLE I. Kinetics prefactor and steady-state connectivity obtained for various volume fraction. The confidence intervals (one standard deviation) are reported between parenthesis.

\begin{tabular}{lllll}
\hline \hline$\rho$ & $37.5 \%$ & $40 \%$ & $45 \%$ & $50 \%$ \\
\hline$D( \pm 2)$ & 171.3 & 163.9 & 158.3 & 156 \\
connectivity $( \pm 0.05)$ & 1.14 & 1.29 & 1.43 & 1.50 \\
\hline \hline
\end{tabular}

time evolution of the characteristic length scale follows the expected power-law as depicted in Fig. 2(b). In this steadystate regime, the length scale evolution can be expressed as $\lambda(t)^{4}=\lambda_{t=0}^{4}+D M t$, where $D$ is a dimensionless coefficient that depends slightly on the volume fraction and is reported in Table I. Thus knowing the mobility coefficient $M$ for a given material would enable to predict the coarsening rate of the structure; or reciprocally, measurements obtained from experimental observations [43] could allow to extract a quantitative estimate of the mobility $M$.

Other valuable information about the structure evolution can be extracted from the interface morphology characterized by the interface curvatures $\left(\kappa_{1}, \kappa_{2}\right)$. These quantities are evaluated on every point of the interface from the phase-field function following Ref. [32]. These quantities are commonly displayed in the form of the probability density function for a point of the interface to have curvature $\left(\kappa_{1}, \kappa_{2}\right)$. Such interface shape distribution (ISD) are shown in Fig. 3 for various volume fractions. To assess the self-similarity behavior of the coarsening, the curvatures are rescaled by the characteristic length scale $\lambda$. The left and right columns display respectively ISD obtained at short $\left(t=240 W^{4} / M\right)$ and long simulation times $\left(t=6000 W^{4} / M\right)$. As expected for this type of connected microstructures, the vast majority of the interface displays curvatures of opposite signs, characteristic of locally saddle shape morphologies.

Let us first examine the ISD obtained for $50 \%$ volume fraction [Figs. 3(a) and 3(b)]. Both ISD are symmetric with respect to $\kappa_{2}=-\kappa_{1}$ dash line, as expected for this volume fraction that has necessarily the same amount of concave and convex surfaces.

The influence of volume fraction is also clearly visible in Fig. 3. While the ISD obtained for $\rho=50 \%$ are symmetric with respect to $\kappa_{2}=-\kappa_{1}$ line, it is no longer the case for lower volume fractions that contain less saddle-shape interfaces and more interfaces with $\kappa_{1}$ and $\kappa_{2}$ of the same sign (i.e., locally convex shape). This is also consistent with the results obtained in experiments $[27,43]$ and in phase-field simulations for bulkdiffusion coarsening [30-32].

However, we note a slight difference between ISD obtained at short and long time: the probability density distribution obtained at longer times appears more concentrated and less spread out in the $\kappa_{2}=\kappa_{1}$ direction.

To check this more thoroughly, we show in Figs. 4(a) and 4 (b) the probability density function of the rescaled average curvature $H \lambda=\lambda\left(\kappa_{1}+\kappa_{2}\right) / 2$ for two different times and volume fractions. This function can be seen as the projection of the ISD on the $\kappa_{1}=\kappa_{2}$ dash line. For both volume fractions, we clearly see that the maximum of the probability function increases with time while the tails of the distribution diminish slightly. This is also shown in Fig. 4(c) where the decrease of 

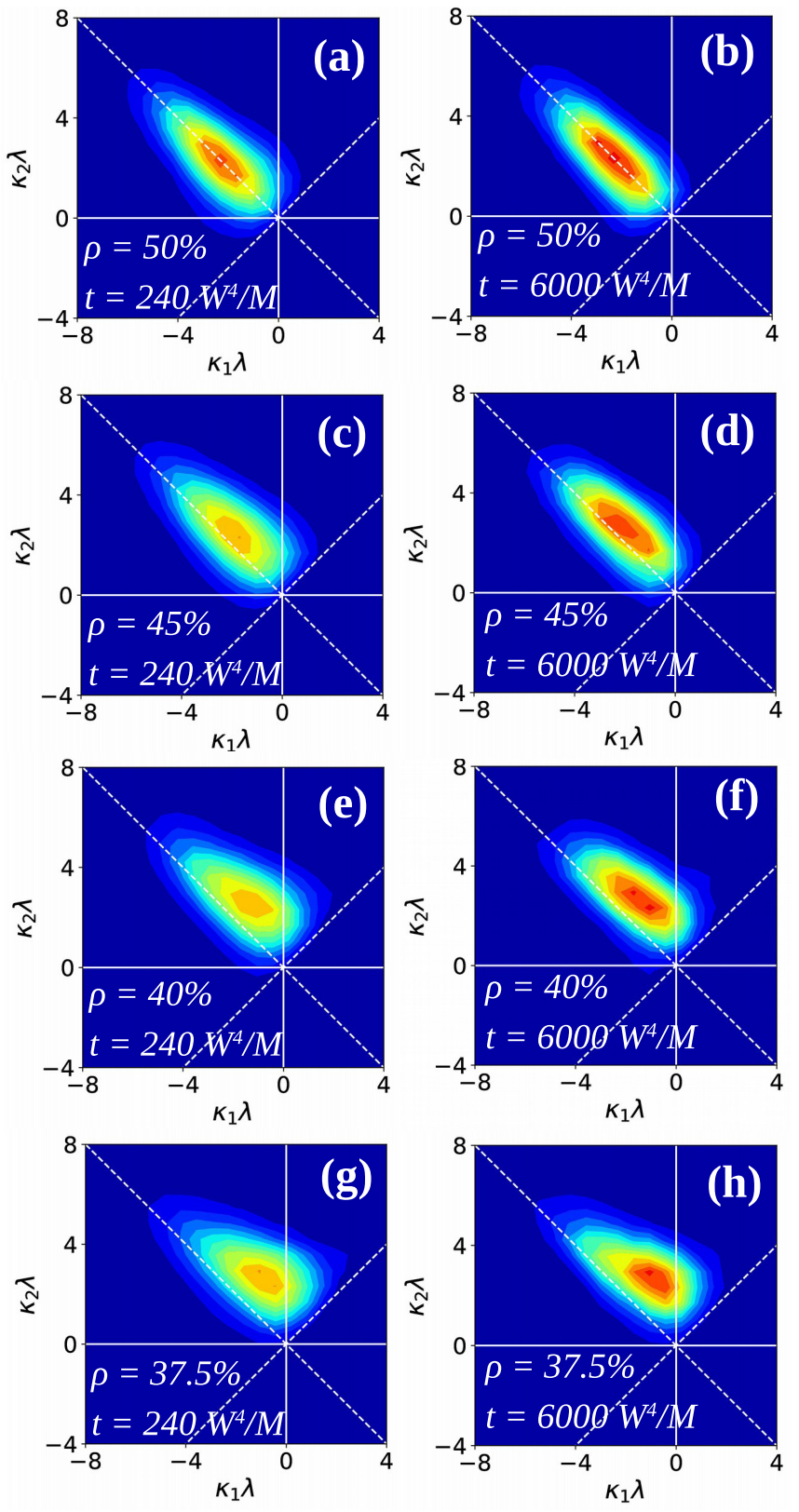

FIG. 3. ISD obtained for the different volume fractions at the short times $t=240 W^{4} / M$ (left column) and long simulation times $t=6000 W^{4} / M$ (right column).

the distribution standard deviation is visible, especially for the low volume fraction $\rho=37.5 \%$.

This can be attributed to the different scaling laws attributed to surface and bulk coarsening. Indeed, the initial microstructures are taken from spinodally decomposed structures that have evolved by bulk diffusion. The ISD obtained after short times still display features characteristics of this regime. In particular, for this bulk-diffusion dynamics, a perturbation of length scale $\lambda$ is smoothed out on a time scaling like $t_{v} \sim \lambda^{3}$. In contrast, for surface diffusion, this time scales like $t_{s} \sim \lambda^{4}$ (see Ref. [33]). Therefore the regions with large average curvatures have higher velocities in the surface diffusion case than in the volume diffusion case, explaining that the ISD are less spread-out after being subjected to surface (a)

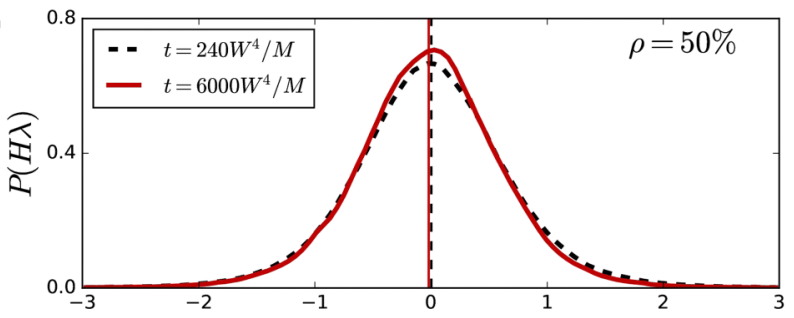

(b)
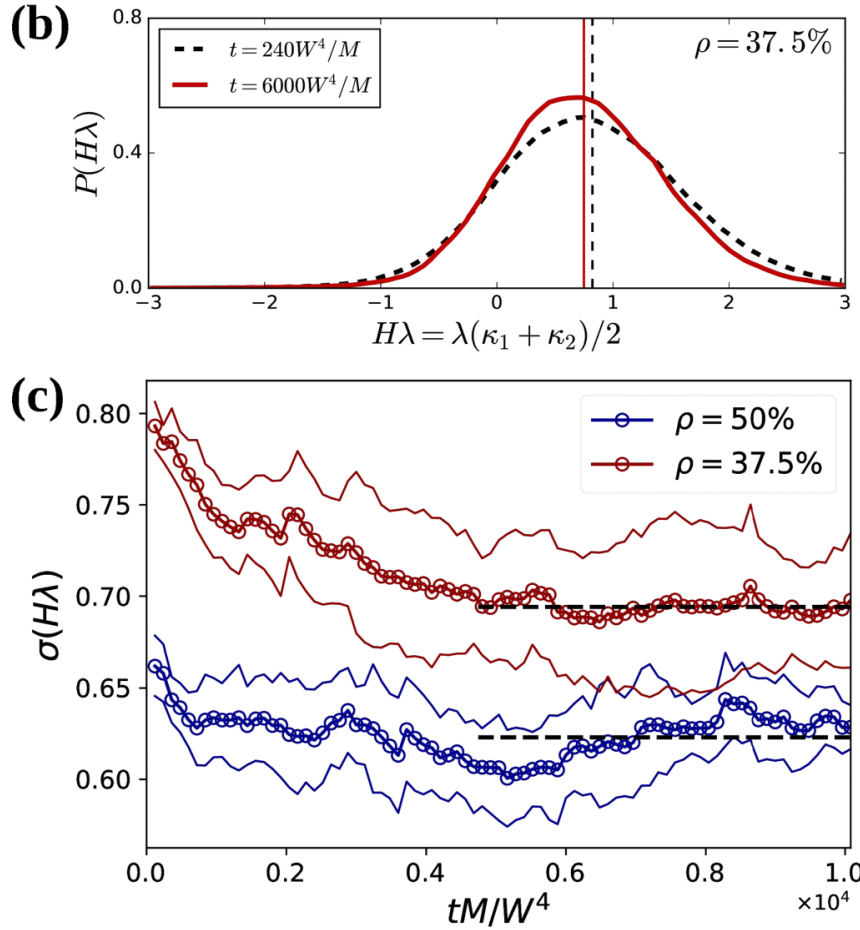

FIG. 4. [(a) and (b)] Probability distribution of the average curvature $H$ rescaled with the characteristic curvature $\lambda$ at two different times and for volume fraction $\rho=50 \%$ and $\rho=37.5 \%$. (c) Temporal evolution of the standard deviation of the probability distribution depicted in (a) and (b). The symbols display the value average over ten independent simulations and the continuous lines a confidence interval.

diffusion coarsening. This shrinkage is even more pronounced for lower volume fractions as displayed for $\rho=37.5 \%$ in Figs. 4(b) and 4(c).

We note that for $\rho=37.5 \%$, this shrinkage occurs on a transient regime with a duration comparable to the transient regime observed in Fig. $2\left(t \lesssim 500 W^{4} / M\right)$. In the following steady-state regime, the ISD evolution can be described as being self-similar: the features of the ISD rescaled by $\lambda$ (average curvatures and the first moments) remain constants or fluctuate slightly within the incertitude interval as displayed in Fig. 4(c).

The slightly different coarsening rates obtained for the different volume fractions and discussed in the previous section can also be analyzed in light of Fig. 4(c). Indeed, the driving force for the coarsening is related to the spreading of the interface distribution along the line $\kappa_{2}=\kappa_{1}$ : matter diffuses from regions of curvature above the average towards regions of curvature below and the intensity of this mass transfer is proportional to the difference between the local curvature and the average curvature of the structure. In other words, 
a large standard deviation of the average curvature leads to a large coarsening rate. Therefore, as shown in Fig. 4(c), the larger value of the standard deviation of $H \lambda$ for $\rho=37.5 \%$ as compared to $\rho=50 \%$ can be related to the difference of coarsening rate observed on Fig. 2.

On the other hand, the mass transport from one part of the structure to another can also be limited by the length of the path between both regions and by the average connectivity of the structure that is directly related to the topology of the structure.

\section{Topological evolution}

Like most of the previous studies, we rely on the genus denoted $g$ to characterize the topology of our microstructures. The genus is often described as the number of handles in a given structure: it is 0 for a sphere, 1 for a torus, etc. It is related to the structure morphology by the Gauss-Bonnet relation:

$$
g=1-\frac{1}{4 \pi} \int_{S} K d A,
$$

where the integral runs over the structure surface and $K=\kappa_{1} \kappa_{2}$ is the local gaussian curvature defined everywhere along the surface. Some previous study used this relation to compute the genus of the structure [28,30-32].

For reasons explained in Appendix B, we preferred a different approach. We first use the marching-cube algorithm [44] to obtain a description of the interface as a set of connected simplexes. Then, we rely on the Euler formula that relates the genus to simple characteristics of this set of simplexes:

$$
g=\frac{1}{2}(2-V+E-F),
$$

where $V, E$, and $F$ are respectively the number of vertices, edges and faces in the set of simplexes. After running the marching cube algorithm on a given structure, the computation of $g$ is then straightforward.

Let us note also that $g$ is not an extensive property of the structure: it is obvious from Eq. (10) that $g$ does not double when the periodic structure is repeated. However, $(g-1)$ (referred hereafter as the connectivity) is extensive in this sense and is therefore the relevant topological quantity when dealing with periodic surfaces [45]. This rectification may not change drastically results when dealing with large structures such as the ones presented in Fig. 1(a) or b where the genus is typically higher than one hundred, but it is important when comparing these structures with minimal surfaces such as the gyroid or the P surface [46], whose genus are of the order of unity per unit cell.

As an example, Fig. 5(a) displays the time evolution of the connectivity for the volume fraction $\rho=50 \%$. We note that during the microstructure evolution, the connectivity decreases monotonically with time, and no "reconnection" is observed. This strictly decreasing evolution is not necessarily obvious for structures evolving through surface diffusion where bridge reconnection can in principle occur in specific situations, such as the collapse of surface pits in the siliconon-nothing technology [47].

Once the connectivity $(g-1)$ is computed for a given structure, it is rescaled with the characteristic volume of the
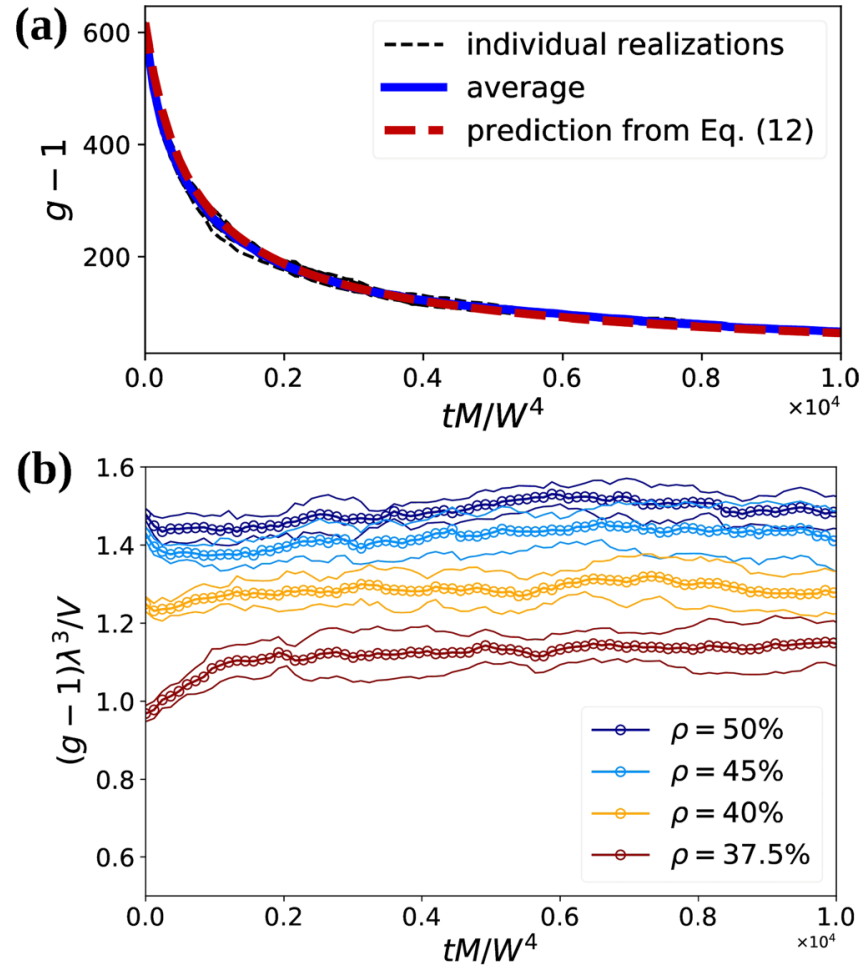

FIG. 5. (a) Time evolution of the connectivity obtained for $\rho=50 \%$. (b) Rescale connectivity as a function of time for different volume fraction. The continuous lines represent confidence interval of one standard deviation.

unit cell $\lambda^{3}$ to obtain the connectivity per unit cell whose evolution is displayed in Fig. 5(b) as a function of time. The average behavior from ten independent simulations is shown with symbols while the standard deviations of these results are displayed with continuous lines. We observe different behaviors for the various volume fractions. In particular, the evolution obtained for the volume fraction $\rho=37.5 \%$ clearly increases before reaching a steady-state value. This transient regime can be related to the transient morphological evolution discussed in the previous section. After this transient regime, the rescale connectivity remains constant for all volume fractions, showing that the topological evolution of the structures is self-similar. This steady-state value of the rescaled connectivity (reported in Table I) increases with volume fraction and reach a maximum of $1.50 \pm 0.05$ for $\rho=50 \%$.

We note that during the time evolution of the structures, the characteristic length scale evolves like $\lambda^{4}=\lambda_{0}^{4}+D M t$. Therefore, since the rescaled connectivity remains constant $($ denoted $C$ ) in the self-similar regime, we necessarily have

$$
g(t)-1=\frac{C V}{\left(\lambda_{0}^{4}+D M t\right)^{3 / 4}} .
$$

As expected, this prediction drawn with a red dash line in Fig. 5(a) reproduces very well the time evolution of the connectivity.

We can also compare the rescaled connectivity of these structures with the one of well-know minimal surfaces [46]. The gyroid, sometimes considered as a model arrangement for these complex connected structures has a rescaled 
connectivity of 4 , well above the values obtained here. A similar result was obtained by Kwon et al. [30-32] for structures evolved by bulk diffusion. Therefore the topology of the structures coarsened by surface diffusion are very different than the gyroid surface which is much more connected. The minimal surface with the lowest connectivity is the Schwartz $\mathrm{P}$ surface that has a rescaled connectivity of 2 , still significantly above the values obtained here.

\section{INFLUENCE OF SURFACE ANISOTROPY}

The results presented in the previous sections are obtained for surfaces with isotropic surface energy and mobility. However, experimental observations on nanoporous gold [26,27] demonstrated that anisotropic surface energy affects significantly the morphology of the structures.

Surface energy anisotropy can be incorporated in a straightforward way in the RRV model. Following the approach described in Ref. [39], we incorporate a weak surface anisotropy of the form

$$
\gamma(\boldsymbol{n})=\bar{\gamma}\left[1+\epsilon_{4}\left(5\left(n_{x}^{4}+n_{y}^{4}+n_{z}^{4}\right)-3\right)\right],
$$

where $\bar{\gamma}$ is the surface energy averaged over all orientations and $\epsilon_{4}$ is the anisotropy parameter. This expression displays a quadratic symmetry representative of anisotropy for facecentered and body-centered structures. Also, we restrict ourselves to weak surface energy anisotropy that satisfy $\epsilon_{4}<1 / 18$ in order to avoid any singular orientations. In principle, stronger anisotropy could also be investigated in the RRV formalism but would necessitate the development of more complex numerical tools $[39,48,49]$.

Microstructures obtained for $\rho=50 \%$ after a time $t M / W^{4}=1200$ are shown in Figs. 6(a) and 6(b) for $\epsilon_{4}=0$ and 0.05 , respectively. We can already see that Fig. 6(b) displays less isotropic microstrcuture with more surfaces oriented along the [111] directions that have the lowest energy [see Eq. (13)]. The anisotropic morphology is more visible on Figs. 6(c) and 6(d) that displays the interfacial normal distribution in a polar plot. We clearly see that in the anisotropic case [Fig. 6(d)], the [111] normal directions is over-represented as compared to [100] or [110] that have higher energies. In contrast, Fig. 6(c) obtained in the isotropic case does not display any privileged orientation.

However, this clear influence of the surface anisotropy on the structure morphology does not affect significantly the coarsening kinetics of the structure as shown in Fig. 6(e) that displays the time evolution of the characteristic length scale for both isotropic and anisotropic cases. A strong surface anisotropy influences the surface orientation but does not change significantly the chemical potential along the interface that control the coarsening kinetics.

The difference of surface anisotropy leads to topologically different microstructures as pointed out by dashed circles in Figs. 6(a) and 6(b). This is expected because of the influence of surface anisotropy on the dynamics of the Rayleigh-Plateau instability which is slowed down or accelerated depending on crystalline orientation of the ligament [50]. However, as shown in Fig. 6(e), the average rescaled connectivity is not significantly affected by the surface anisotropy and fluctuates around the value 1.5 obtained for isotropic structures.
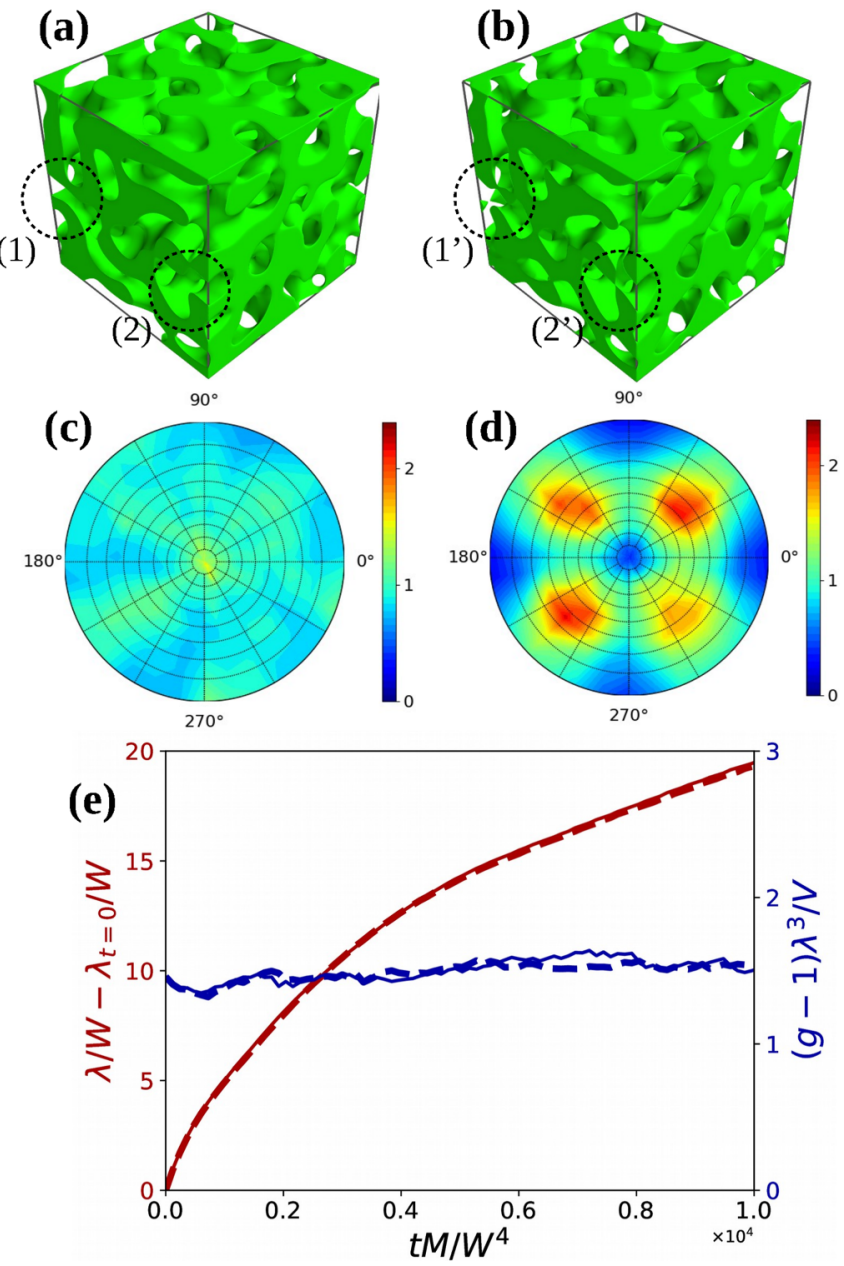

FIG. 6. [(a) and (b)] Structures obtained at $t M / W^{4}=1200$ without (a) and with (b) interface anisotropy. [(c) and (d)] Density of surface normal without (c) and with (d) interface anisotropy. (e) Time evolution of the characteristic length scale and rescaled connectivity for both isotropic (continuous lines) and anisotropic structures (dash lines).

Similar results are obtained for the other volume fractions: despite the influence of the surface anisotropy on the structure morphology, it affects only marginally the coarsening kinetics and the evolution of the rescaled connectivity. However, we note that a stronger surface anisotropy for which some orientations would be forbidden could influence significantly the coarsening kinetics by completely impeding the development of the Rayleigh-Plateau instability and ligament pinch-off in some directions.

\section{COMPARISON WITH EXPERIMENTAL MICROSTRUCTURES}

In addition to the numerical study presented above, we propose here to compare our results with tomographic pictures of samples obtained from liquid metal dealloying [51]. As detailed in Ref. [51], two different samples with volume fractions of $40 \%$ and $50 \%$ have been obtained from precursor alloys of $(\mathrm{FeCr})_{0.4} \mathrm{Ni}_{0.6}$ and $(\mathrm{FeCr})_{0.5} \mathrm{Ni}_{0.5}$ compositions respectively. Upon immersion of the samples in liquid $\mathrm{Mg}$ during 1 hour at $1097 \mathrm{~K}, \mathrm{Ni}$ is dissolved selectively in the $\mathrm{Mg}$ 
(a)
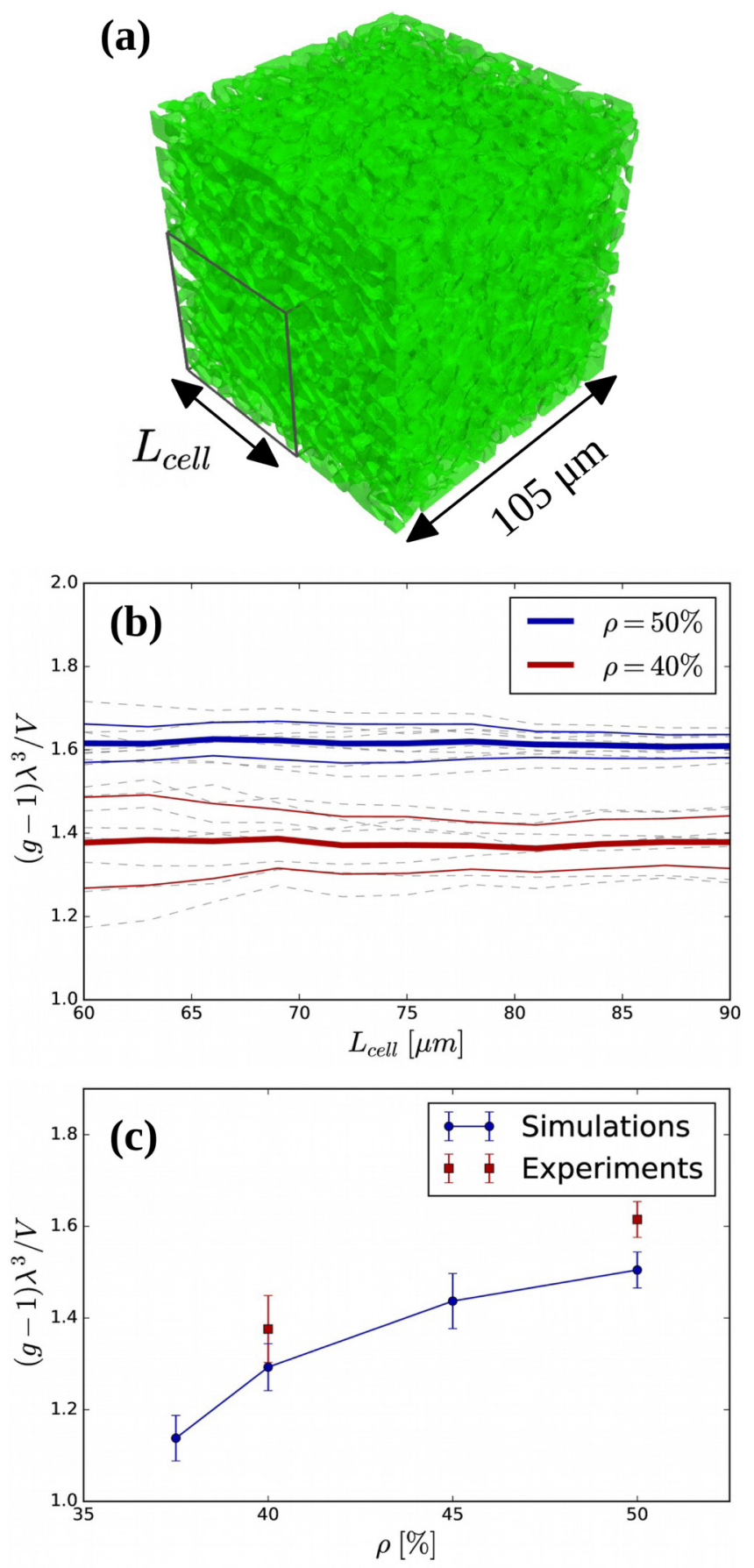

FIG. 7. (a) Tomographic imaging of a microporous FeCr sample with volume fraction $50 \%$ obtained from liquid metal dealloying [51]. (b) Rescaled connectivity as a function of the size of the analyzed volume (see text for explanations). (c) Comparison of the average connectivity with numerical results.

bath while $\mathrm{Fe}$ and $\mathrm{Cr}$ reorganize and form a porous connected structure. The 3D microstructure of the samples are obtained using $\mathrm{x}$-ray tomography, leading to observations as the one shown in Fig. 7(a) for a volume fraction of $50 \%$. We note that, in these experiments, the time necessary to dealloy the sample thickness is small ( $\lesssim 10 \mathrm{~min})$ compared to the subsequent coarsening ( $\sim 50 \mathrm{~min})$ [52]. During this coarsening stage, the ligament size grows significantly from about $400 \mathrm{~nm}$ to $4 \mu \mathrm{m}$.
In addition, these changes are expected to takes place by surface diffusion because of the strong immiscibility of $\mathrm{Fe}$ and $\mathrm{Cr}$ in liquid $\mathrm{Mg}$ and the slow diffusion in the solid phase [14].

From the microstructure shown in Fig. 7, the rescaled connectivity can be obtained using the analysis discussed above. We first compute the characteristic length scale of both structures (40\% and $50 \%$ volume fractions) with the structure factor analysis and find characteristic length scales of $\lambda_{40 \%}=8.43 \mu \mathrm{m}$ and $\lambda_{50 \%}=7.68 \mu \mathrm{m}$, respectively. We note that these quantities are in good agreement with the sum of the ligament and pore size reported in Ref. [51].

Considering the finite size of the samples, the structures topology can be more challenging to measure precisely. To assess the influence of surface effects on the measure of the structure connectivity, we analyze various volumes of different sizes that are representative subsets of the global structure [such as the one of dimension $L_{\text {cell }}$ shown in Fig. 7(a)]. To be consistent with the analysis of numerical structures, the cell is replicated eight times with mirror boundary conditions in order to obtain a periodic structure. The genus of this periodic structure is then computed based on the Euler formula of Eq. (11). Figure 7(b) displays the rescaled connectivity as a function of cell sizes (all cells are cubic). The dashed grey lines represent the rescaled connectivity for different locations of the subcell (eight different locations are considered, each of them corresponding to a corner of the structure) and the thick lines show the evolution of their average (thin continuous lines represents the standard deviations).

The average rescaled connectivity does not vary significantly with the cell size, which shows that the volumes are large enough to be representative and that the artifacts introduced by replicating the structure do not influence significantly the results. We obtain values of the rescaled connectivity of $1.38 \pm 0.08$ and $1.62 \pm 0.04$, respectively, for $40 \%$ and $50 \%$. As shown in Fig. 7(c), these estimates fall close (within $10 \%$ ) to the values obtained from numerical microstructures, showing a good agreement between numerical and experimental microstructures.

A discrepancy between numerical and experimental structures is expected because the microstructures obtained numerically are perfectly isotropic while the samples obtained from liquid metal dealloying present a privileged orientation aligned with the dealloying direction.

\section{CONCLUSION}

We have used a phase-field model to characterize the evolution of connected structures by surface diffusion, that is ubiquitous during the fabrication of nano/micro-porous materials with the dealloying technique. We show that after a transient regime, the evolution of the characteristic length scale of these microstructures follows the expected $t^{1 / 4}$ powerlaw for the different volume fractions investigated here. In addition, in this steady-state regime, we find that the structures evolve in a self-similar way: both morphological and topological characteristics remain constant once rescaled by the characteristic length scale. This contrasts with the conclusion of previous experimental and numerical studies [27,28] that showed a lack of self-similarity of coarsening by surface diffusion. 
For the atomistic study of Erlebacher [28], this discrepancy can be related to two notable differences with our simulations: first, Erlebacher considered finite-size particles in contrast with the infinite periodic systems investigated here. The incorporation of a second length scale (the radius of the particle) can influence significantly the coarsening behavior and break down its self-similar evolution. Second, the microstructures of Ref. [28] are obtained after a dealloying step that consists in removing progressively atoms from an initial binary structure. Therefore the topological and morphological characteristics of these microstructures might differ significantly from the ones presented here that are obtained by spinodal decomposition, as suggested in Ref. [53]. These differences might influence the subsequent coarsening mechanisms and its selfsimilar character.

A lack of self-similarity is also mentioned in Ref. [27], on the basis of tomographic observations of nanoporous gold. It is suggested that the non-self-similar character of the coarsening is related to the surface energy anisotropy. As discussed in Sec. IV, we show that a weak surface anisotropy (without singular orientations) does not change significantly the coarsening rate and the self-similar behavior of the coarsening. However, in the case of nanoporous gold evolution at moderate temperatures $\left(550{ }^{\circ} \mathrm{C}-650^{\circ} \mathrm{C}\right.$ in the case of Ref. [27]), the magnitude of the surface energy anisotropy might be stronger than investigated here, impeding the development of the Rayleigh-Plateau instabilities along some crystalline orientations, and breaking down self-similar coarsening.

The above discussion reveals the limitations of the current study and hints perspectives for future works. A first limitation lies in the choice of the initial structure obtained from spinodal decomposition. Indeed, in the context of structures obtained from dealloying, the dealloying direction is always a privileged orientation of the system, making the resulting structure anisotropic, even after significant coarsening. In addition, it was suggested that structures manufactured from dealloying can present significantly different topologies than the ones obtained from spinodal decomposition [53]. To account for these effects, a natural extension of this work will consist of taking more realistic initial microstructures, obtained either from numerical models $[1,16,28]$ or from tomographic observations of dealloyed samples [26,27,43,51]. Another limitation of the present study is the weak surface energy anisotropy considered in Sec. IV that may not represent the case of nanoporous gold [5,27] or other porous materials. Future work will consist in extending the RRV model used in this study to account for such strong anisotropy $[48,49]$ to investigate the coarsening behavior in this situation.

\section{ACKNOWLEDGMENTS}

The authors would like to gratefully thank Morgane Mokhtari, Christophe Le Bourlot, and Eric Maire for supplying the $\mathrm{x}$-ray tomographic structures discussed in Sec. V. P.A.G. would also like to personally thank Tetsuo Mohri (IMR, Tohoku Univesrity) for fruitful discussion on this work. Also P.A.G. gratefully acknowledges SR16000 supercomputing resources from the Center for Computational Materials
Science of the Institute for Materials Research, Tohoku University.

\section{APPENDIX A: CHECK OF SURFACE DIFFUSION MODEL}

To check the numerical implementation of the model, we consider the simple case of an interface with an initially perturbed position $h(x)=h_{0} \sin \left(2 \pi x / L_{x}\right)$. Figure $8(\mathrm{~b})$ shows the time evolution of the perturbation amplitude: as expected by the sharp-interface solution in the limit $h_{0} \ll L_{x}$, the amplitude of this perturbation decreases exponentially in time, following $h(t)=h_{0} e^{\omega_{k} t}$ with a decay rate $\omega_{k}=-M k^{4}$ reported in Fig. 8(c). This power law with an exponent $n=4$ is ubiquitous in surface diffusion problems [33]. The slight

(a)

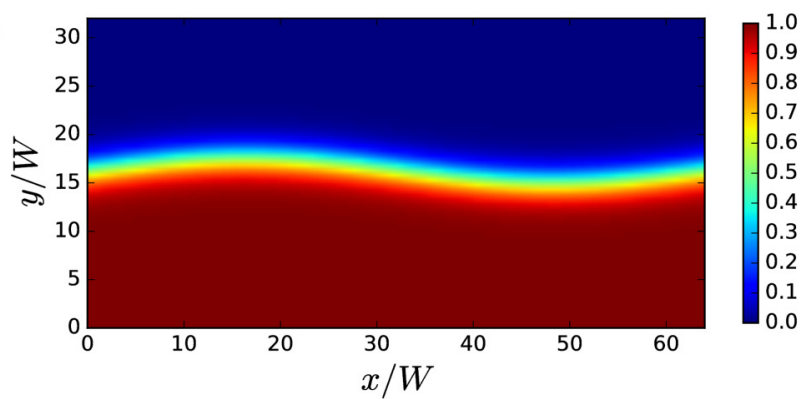

(b)
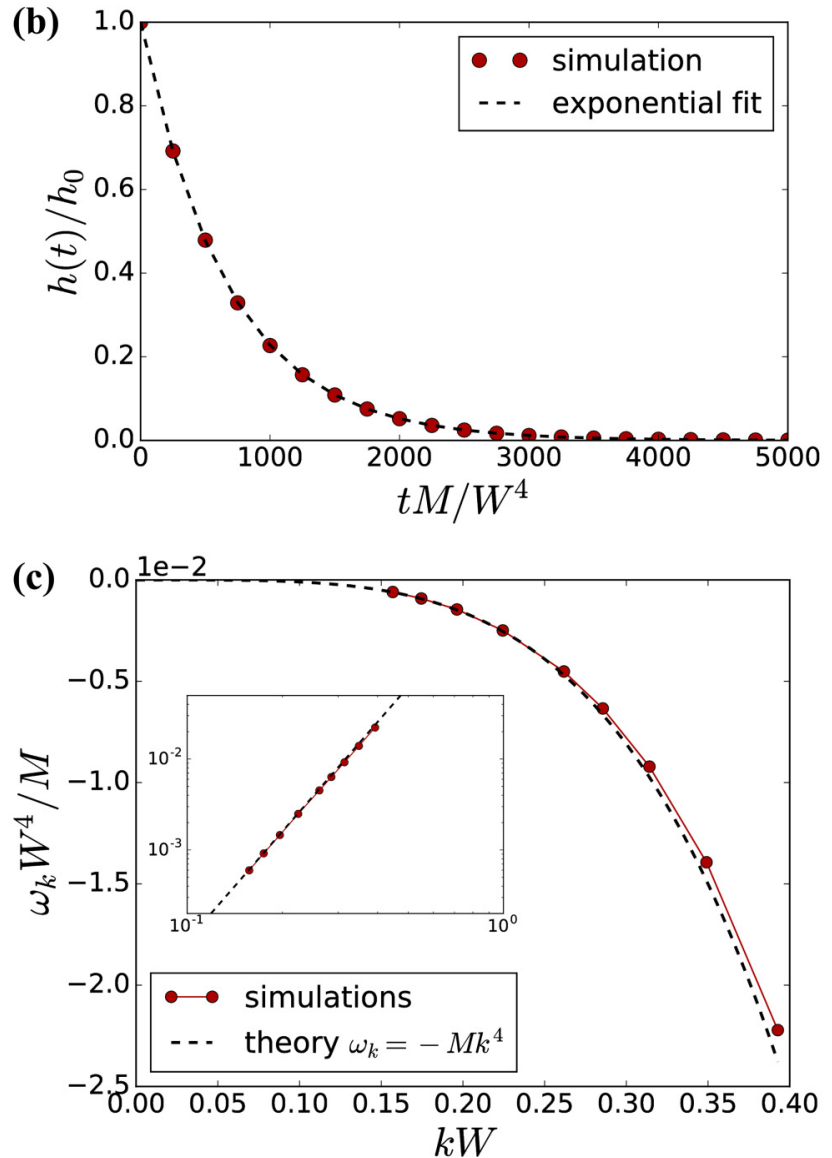

FIG. 8. (a) Slightly perturbed interface. (b) Exponential relaxation kinetics to equilibrium. (c) Growth rate as a function of wave vector of the perturbation, showing that dispersion relation $\omega_{k}=$ $-M k^{4}$ is well verified. 


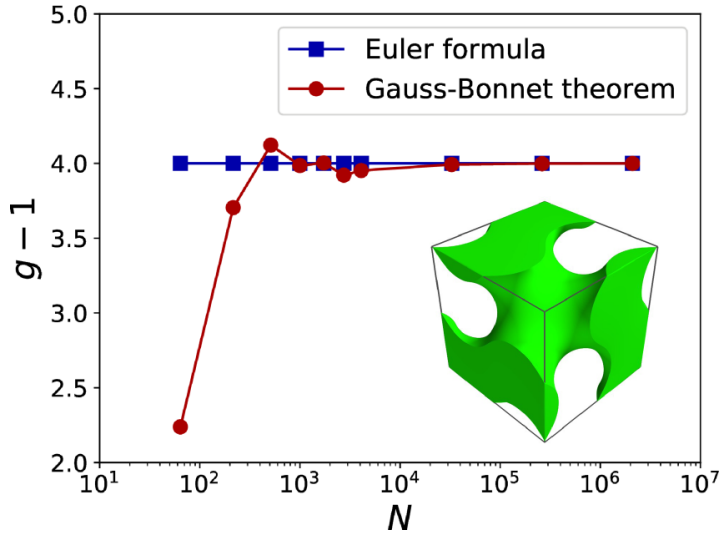

FIG. 9. Connectivity $(g-1)$ obtained from integration of the Gauss-Bonnet integral and from Euler formula for the gyroid structure as a function of the number of voxels in the system.

deviation (less than 5\%) obtained for short wave-length is attributed to the numerical discretization of the model.

\section{APPENDIX B: COMPUTATION OF THE TOPOLOGICAL CHARACTERISTICS OF THE STRUCTURES}

Several approaches can be used to compute the topological characteristics (i.e., the genus) of structures investigated here. In particular, some previous studies relied on the GaussBonnet relation [Eq. (10)] to compute the genus from the integration of the curvature along the surface. While this method is mathematically valid, its practical implementation on a discretized structure can present some difficulties. First, the computation of the Gaussian curvature must rely on a field whose evolution is slow compared to the grid spacing [30-32]. In addition, once an estimate of the gaussian curvature is known, it needs to be interpolated on the surface before estimating the integral in Eq. (10). These additional steps introduce small numerical errors, especially for curvature radii close to the grid spacing.

The influence of these numerical errors is demonstrated in Fig. 9 where the computed connectivity of the gyroid structure is shown as function of the number of voxels in the system. For fine discretizations of the structure, the connectivity converges towards the expected value of $g-1=4$. However, for coarser discretizations, the obtained value of the connectivity diverts significantly from 4 due to the accumulation of numerical errors. Especially, for the coarser grid of $4 \times 4 \times 4$ voxels, the integration of the Gauss-Bonnet relation entirely fails to give the correct value of the connectivity.

As discussed in the main paper, we prefer to rely on the Euler formula to compute these topological quantity. After applying a marching cube algorithm to the structure, we compute the genus by using the Euler relation of Eq. (11). The result is thus immediately an integer value and does not present any computational challenge. As illustrated in Fig. 9, using the Euler formula provides a better numerical stability even for a very coarse numerical grids.
[1] J. Erlebacher, M. Aziz, A. Karma, N. Dimitrov, and K. Sieradzki, Nature (London) 410, 450 (2001).

[2] D. Kramer, R. N. Viswanath, and J. Weissmüller, Nano Lett. 4, 793 (2004).

[3] J. Biener, A. Wittstock, L. Zepeda-Ruiz, M. Biener, V. Zielasek, D. Kramer, R. Viswanath, J. Weissmüller, M. Bäumer, and A. Hamza, Nat. Mater. 8, 47 (2009).

[4] A. Wittstock, V. Zielasek, J. Biener, C. Friend, and M. Bäumer, Science 327, 319 (2010).

[5] T. Fujita, P. Guan, K. McKenna, X. Lang, A. Hirata, L. Zhang, T. Tokunaga, S. Arai, Y. Yamamoto, N. Tanaka et al., Nat. Mater. 11, 775 (2012).

[6] J. Biener, A. Hodge, J. Hayes, C. Volkert, L. Zepeda-Ruiz, A. Hamza, and F. Abraham, Nano Lett. 6, 2379 (2006).

[7] X.-Y. Sun, G.-K. Xu, X. Li, X.-Q. Feng, and H. Gao, J. Appl. Phys. 113, 023505 (2013).

[8] E. Bringa, J. Monk, A. Caro, A. Misra, L. Zepeda-Ruiz, M. Duchaineau, F. Abraham, M. Nastasi, S. Picraux, Y. Wang et al., Nano Lett. 12, 3351 (2011).

[9] M. Hakamada and M. Mabuchi, J. Alloy. Compd. 479, 326 (2009).

[10] Z. Zhang, Y. Wang, Z. Qi, W. Zhang, J. Qin, and J. Frenzel, J. Phys. Chem. C. 113, 12629 (2009).

[11] L.-Y. Chen, J.-S. Yu, T. Fujita, and M.-W. Chen, Adv. Func. Mater. 19, 1221 (2009).

[12] T. Wada, K. Yubuta, A. Inoue, and H. Kato, Mater. Lett. 65, 1076 (2011).
[13] M. Tsuda, T. Wada, and H. Kato, J. Appl. Phys. 114, 113503 (2013).

[14] T. Wada and H. Kato, Scr. Mater. 68, 723 (2013).

[15] J. Kim, M. Tsuda, T. Wada, K. Yubuta, S. Kim, and H. Kato, Acta Mater. 84, 497 (2015).

[16] P.-A. Geslin, I. McCue, B. Gaskey, J. Erlebacher, and A. Karma, Nat. Commun. 6, 8887 (2015).

[17] I. McCue, B. Gaskey, P.-A. Geslin, A. Karma, and J. Erlebacher, Acta Mater. 115, 10 (2016).

[18] T. Wada, T. Ichitsubo, K. Yubuta, H. Segawa, H. Yoshida, and H. Kato, Nano Lett. 14, 4505 (2014).

[19] Y. Sun, Y. Ren, and K. Yang, Mater. Lett. 165, 1 (2016).

[20] Z. Lu, C. Li, J. Han, F. Zhang, P. Liu, H. Wang, Z. Wang, C. Cheng, L. Chen, A. Hirata et al., Nat. Commun. 9, 276 (2018).

[21] Y. Ding, Y.-J. Kim, and J. Erlebacher, Adv. Mater. 16, 1897 (2004).

[22] L. Qian and M. Chen, Appl. Phys. Lett. 91, 083105 (2007).

[23] Q. Chen and K. Sieradzki, Nat. Mater. 12, 1102 (2013).

[24] J. Clum, Scr. Metall. 4, 463 (1970).

[25] J. Snyder, P. Asanithi, A. Dalton, and J. Erlebacher, Adv. Mater. 20, 4883 (2008).

[26] Y.-C. K. Chen, Y. Chu, J. Yi, I. McNulty, Q. Shen, P. Voorhees, and D. Dunand, App. Phys. Lett. 96, 043122 (2010).

[27] Y.-C. K. Chen-Wiegart, S. Wang, Y. Chu, W. Liu, I. McNulty, P. W. Voorhees, and D. Dunand, Acta Mater. 60, 4972 (2012).

[28] J. Erlebacher, Phys. Rev. Lett. 106, 225504 (2011).

[29] K. Kolluri and M. Demkowicz, Acta Mater. 59, 7645 (2011). 
[30] Y. Kwon, K. Thornton, and P. W. Voorhees, Phys. Rev. E 75, 021120 (2007).

[31] Y. Kwon, K. Thornton, and P. Voorhees, Europhys. Lett. 86, 46005 (2009).

[32] Y. Kwon, K. Thornton, and P. Voorhees, Philos. Mag. 90, 317 (2010).

[33] C. Herring, J. Appl. Phys. 21, 301 (1950).

[34] A. M. Lacasta, A. Hernández-Machado, J. M. Sancho, and R. Toral, Phys. Rev. B 45, 5276 (1992).

[35] J. Zhu, L.-Q. Chen, J. Shen, and V. Tikare, Phys. Rev. E 60, 3564 (1999).

[36] D.-H. Yeon, P.-R. Cha, and M. Grant, Acta Mater. 54, 1623 (2006).

[37] C. Gugenberger, R. Spatschek, and K. Kassner, Phys. Rev. E 78, 016703 (2008).

[38] A. A. Lee, A. Münch, and E. Süli, App. Phys. Lett. 107, 081603 (2015).

[39] A. Rätz, A. Ribalta, and A. Voigt, J. Comput. Phys. 214, 187 (2006).

[40] W. Mullins, J. Appl. Phys. 30, 77 (1959).

[41] H. Wong, M. J. Miksis, P. W. Voorhees, and S. H. Davis, Scr. Mater. 39, 55 (1998).

[42] T. Fujita and M. Chen, Jpn. J. Appl. Phys. 47, 1161 (2008).
[43] Y.-C. K. Chen-Wiegart, T. Wada, N. Butakov, X. Xiao, F. De Carlo, H. Kato, J. Wang, D. Dunand, and E. Maire, J. Mater. Res. 28, 2444 (2013).

[44] W. Lorensen and H. Cline, in ACM Siggraph Computer Graphics (ACM Press, New York, 1987), Vol. 21, pp. 163-169.

[45] We note that the Euler characteristic $\chi=2(1-g)$ is also a valid extensive quantity.

[46] W. T. Góźdź and R. Hołyst, Phys. Rev. E 54, 5012 (1996).

[47] I. Mizushima, T. Sato, S. Taniguchi, and Y. Tsunashima, Appl. Phys. Lett. 77, 3290 (2000).

[48] S. Torabi, J. Lowengrub, A. Voigt, and S. Wise, Proc. Roy. Soc. A 465, 1337 (2009).

[49] M. Salvalaglio, R. Bergamaschini, R. Backofen, A. Voigt, F. Montalenti, and L. Miglio, Appl. Surf. Sci. 391, 33 (2017).

[50] K. F. Gurski and G. McFadden, Proc. Roy. Soc. A 459, 2575 (2003).

[51] M. Mokhtari, C. Le Bourlot, F. Adrien, A. Bonnin, T. Wada, J. Duchet-Rumeau, H. Kato, and E. Maire, Mater. Charact. 144, 166 (2018)

[52] M. Mokhtari, Ph.D. thesis, Université de Lyon (Fr); Tōhoku University (Jp) (2018).

[53] K. Mangipudi, E. Epler, and C. Volkert, Acta Mater. 140, 337 (2017). 